\title{
COMPLEXITY AND ALGORITHMS FOR COMPUTING VORONOI CELLS OF LATTICES
}

\author{
MATHIEU DUTOUR SIKIRIĆ, ACHILL SCHÜRMANN, AND FRANK VALLENTIN
}

\begin{abstract}
In this paper we are concerned with finding the vertices of the Voronoi cell of a Euclidean lattice. Given a basis of a lattice, we prove that computing the number of vertices is a \# P-hard problem. On the other hand, we describe an algorithm for this problem which is especially suited for lowdimensional (say dimensions at most 12) and for highly-symmetric lattices. We use our implementation, which drastically outperforms those of current computer algebra systems, to find the vertices of Voronoi cells and quantizer constants of some prominent lattices.
\end{abstract}

\section{INTRODUCTION}

Let $L=B \mathbb{Z}^{m} \subseteq \mathbb{R}^{n}$ be a lattice of rank $m$ in Euclidean space given by a matrix $B \in \mathbb{R}^{n \times m}$ of rank $m$. By lin $L$ we denote the linear subspace spanned by the elements of $L$. The Voronoi cell of $L$ is

$$
\mathrm{V}(L)=\{x \in \operatorname{lin} L:\|x\| \leq\|x-v\| \text { for all } v \in L\} .
$$

The Voronoi cell of a lattice is a centrally symmetric, convex polytope. The polytopes $V(L)+v$ for $v \in L$ tile lin $L$. The study of Voronoi cells is motivated by the fact that most important geometric lattice parameters have a direct interpretation in terms of the Voronoi cell: The determinant $\operatorname{det} L$ equals the volume of $\mathrm{V}(L)$, the packing radius $\lambda(L)$ equals the inradius of $\mathrm{V}(L)$, the covering radius $\mu(L)$ equals the circumradius of $\mathrm{V}(L)$, and the quantizer constant $G(L)$ is

$$
G(L)=(\operatorname{det} L)^{-(1+2 / n)} \int_{\mathrm{V}(L)}\|x\|^{2} d x .
$$

Received by the editor April 7, 2008 and, in revised form, August 19, 2008.

2000 Mathematics Subject Classification. Primary 03D15, 11H56, 11H06, 11E10, 52B55, $52 \mathrm{~B} 12$.

Key words and phrases. Lattice, Voronoi cell, Delone cell, covering radius, quantizer constant, lattice isomorphism problem, zonotope.

The work of the first author was supported by the Croatian Ministry of Science, Education and Sport under contract 098-0982705-2707.

The second and third authors were supported by the Deutsche Forschungsgemeinschaft (DFG) under grant SCHU 1503/4-2.

The third author was also supported by the Netherlands Organization for Scientific Research under grant NWO 639.032.203.

All three authors thank the Hausdorff Research Institute for Mathematics for its hospitality and support.

(C)2009 American Mathematical Society Reverts to public domain 28 years from publication 
In this paper we consider theoretical and practical aspects of the computation of the covering radius as well as the quantizer constant of a lattice. These two parameters have many applications, we just name a few: By computing the covering radius, we get an upper bound for the lattice sphere covering problem, which is the problem of minimizing the covering radius among the lattices of fixed determinant (see CS99, Chapter 2] and SV06]). The computation of the covering radius of the Leech lattice in CS99, Chapter 23] had a major impact on the study of hyperbolic reflection groups (see [CS99, Chapter 27]). An upper bound for the Frobenius number of a set of integers can be obtained from the covering radius of a suitable lattice (see [FR05]). A recent application comes from public key cryptography; Micciancio Mic04 found a new connection between the average-case complexity of finding the packing radius and the worst-case complexity of determining the covering radius. In information theory, the quality of a lattice as a vector quantizer is determined by its quantizer constant (see GG92, ELZ05, SB03 and CS99, Chapter 2.3, Chapter 21]).

The structure of this paper is as follows. In Section 2 we discuss the computational complexity of the covering radius problem. We prove that the related problem of counting vertices of the Voronoi cell is \# P-hard. As a byproduct of our construction, we show that the lattice isomorphism problem is at least as difficult as the graph isomorphism problem. We turn to practical algorithms for the covering radius problem in Section 3 . There we describe an algorithm which computes the vertices of the Voronoi cell of a lattice. Based on this algorithm we give an algorithm for computing the quantizer constant in Section 4. In Section 5] we report on computations with our implementation. We determine the exact covering radius and quantizer constants of many prominent lattices which were not known before.

\section{Computational complexity}

We formulate the covering radius problem as a decision problem.

Problem 1. Covering radius problem

Input: $m, n, B \in \mathbb{Q}^{m \times n}, \mu \in \mathbb{Q}$.

Output: Yes, if $\mu\left(B \mathbb{Z}^{n}\right) \leq \mu$, No otherwise.

It is conjectured (see Mic04, Section 1.1]) that the covering radius problem is NP-hard. Haviv and Regev HR06 showed that there is a constant $c_{p}$ so that the covering radius in the $l_{p}$-norm is $\Pi_{2}$-hard to approximate within a constant less than $c_{p}$ for any large enough $p$. In GMR05 Guruswami, Micciancio and Regev proved that approximating it within a factor of $O(\sqrt{m / \log m})$ for a lattice of rank $m$ cannot be NP-hard unless the polynomial hierarchy collapses.

Currently, there is only one known general and practical method to compute $\mu(L)$ for a lattice $L$ : First one enumerates the vertices of $\mathrm{V}(L)$ and then one finds the vertex with largest norm. The number of vertices of $\mathrm{V}(L)$ can be as large as $(m+1)$ ! and furthermore, as we show in Theorem 11, even computing this number is \# P-hard.

Problem 2. Vertices of a lattice Voronoi cell

Input: $m, n, B \in \mathbb{Q}^{m \times n}$.

Output: Number of vertices of $\mathrm{V}\left(B \mathbb{Z}^{n}\right)$. 
Theorem 1. The problem "Vertices of a lattice Voronoi cell" is \# P-hard.

It will be obvious from the proof that we could restrict the problem to the case $m=n$. We reduce the problem "Acyclic orientations of a graph", which Linial Lin86] showed to be \# P-complete, to Problem 2.

Problem 3. Acyclic orientations of a graph

Input: A graph $G=(V, E)$.

Output: The number of orientations of $G$ with no directed circuit.

The structure of the proof of Theorem 1 is as follows: In Section 2.1 we construct a matrix $B$ with columns indexed by $E$ defining a lattice $L(G)=B \mathbb{Z}^{E}$ from $G$ in polynomial time. Then we show that the vertices of the Voronoi cell of $V(L(G))$ are in bijection with the acyclic orientations of $G$. To establish this bijection we need several intermediate steps. In Section 2.2 we associate to $G$ a hyperplane arrangement $H(G)$ whose chambers are in bijection with the acyclic orientations of $G$. In Section 2.3 we recall that the chambers of a hyperplane arrangement are in bijection with the vertices of a zonotope associated to the hyperplane arrangement. These two steps are standard and we cover them rather briefly. In Section 2.4 we show that the Voronoi cell of $L(G)$ is a zonotope which, up to a linear transformation, is the one associated to the hyperplane arrangement $H(G)$. In Section 2.5. as a byproduct of this construction, we show that the lattice isomorphism problem is at least as difficult as the graph isomorphism problem. Some related complexity results concerning vertex enumeration of polyhedra given by linear inequalities are in KBBEG08, Dy83.

2.1. From graphs to lattices. Let $G=(V, E)$ be a connected graph with vertex set $V=\{1, \ldots, n\}$ and edge set $E$. We consider the following orientation of the edges of $G$ : The head of an edge $e=\{v, w\} \in E$ is $e^{+}=\max \{v, w\}$ and the tail is $e^{-}=\min \{v, w\}$.

Let $T \subseteq E$ be the edge set of a spanning tree of $G$, and let $e \in T$. Deleting $e$ from $T$ divides $T$ into two connected components with vertex sets $T_{e}^{+}$and $T_{e}^{-}$, where $e^{+} \in T_{e}^{+}$and $e^{-} \in T_{e}^{-}$. Define the vector $b_{T, e} \in \mathbb{Z}^{E}$ by

$$
b_{T, e}(f)=\left\{\begin{array}{cl}
1, & \text { if } f^{+} \in T_{e}^{+} \text {and } f^{-} \in T_{e}^{-}, \\
-1, & \text { if } f^{-} \in T_{e}^{+} \text {and } f^{+} \in T_{e}^{-}, \\
0, & \text { otherwise. }
\end{array}\right.
$$

Then

$$
L(G, T)=\left\{\sum_{e \in T} \alpha_{e} b_{T, e}: \alpha_{e} \in \mathbb{Z}\right\} \subseteq \mathbb{Z}^{E}
$$

is a lattice of rank $n-1$.

Proposition 1. Let $T$ and $T^{\prime}$ be spanning trees of $G$. Then, $L(G, T)=L\left(G, T^{\prime}\right)$.

Proof. Since one can connect any two spanning trees by a sequence of transformations of the form $T \leftrightarrow T \backslash\{e\} \cup\{f\}$, it suffices to prove the proposition for $T^{\prime}=T \backslash\{e\} \cup\{f\}$. Let $g \in T^{\prime}$. If $g=f$, then $b_{T^{\prime}, f}= \pm b_{T, e}$. If $g \in T$, then denote by $C$ the cycle containing $e$ and $f$. If $g \notin C$, then $b_{T^{\prime}, g}=b_{T, g}$. The subgraph of $G$ with edge set $T \backslash\{e, g\}$ has three connected components, denoted by $C_{1}, C_{2}$, $C_{3}$. Given $h=\{v, w\} \in E$, the value of $b_{T^{\prime}, g}(h), b_{T, g}(h)$ and $b_{T, e}(h)$ depends only on which connected component $v$ and $w$ belong to. So, in computing $b_{T^{\prime}, g}$, we can 
reduce ourselves to the case when $G$ is the complete graph on $\{1,2,3\}, g=\{1,3\}$, $e=\{1,2\}$ and $f=\{2,3\}$. An easy computation gives $b_{T^{\prime}, g}=b_{T, g}+b_{T, e}$ and so we conclude that $b_{T^{\prime}, g}=b_{T, g}+\alpha b_{T, e}$ with $\alpha \in\{-1,0,+1\}$.

In the following we omit the spanning tree $T$ from the notation $L(G, T)$ and just write $L(G)$. Note that one can find a basis of $L(G)$ given $G$ in polynomial time.

\subsection{From graphs to hyperplane arrangements. A matrix}

$$
V=\left(v_{1}, \ldots, v_{m}\right) \in \mathbb{R}^{n \times m}
$$

with nonzero column vectors $v_{i} \in \mathbb{R}^{n}$ gives an arrangement of hyperplanes

$$
H(V)=\left\{H_{1}, \ldots, H_{m}\right\} \quad \text { with } \quad H_{i}=\left\{c \in \mathbb{R}^{n}: c \cdot v_{i}=0\right\} .
$$

The hyperplane arrangement $H(V)$ divides the space $\mathbb{R}^{n}$ into polyhedral cones, called regions, of different dimensions. The regions are partially ordered by inclusion and full-dimensional regions are called chambers.

To associate a hyperplane arrangement $H(G)$ with $G$ we consider the incidence matrix $D_{G} \in \mathbb{R}^{V \times E}$ of $G$ which is given by

$$
D_{G}(v, e)=\left\{\begin{array}{cl}
1, & \text { if } v=e^{+} \\
-1, & \text { if } v=e^{-}, \\
0, & \text { otherwise. }
\end{array}\right.
$$

Then we define the hyperplane arrangement of $G$ by $H(G)=H\left(D_{G}\right)$.

In GZ83, Lemma 7.1] Greene and Zaslavsky show that the chambers of $H(G)$ are in bijection with the acyclic orientations of $G$ : Let $\vec{E}$ be an acyclic orientation of $E$. Then a chamber of $H(G)$ is given by

$$
\operatorname{Reg}(\vec{E})=\left\{x \in \mathbb{R}^{V}: x_{v}<x_{w} \text { if }(v, w) \in \vec{E}\right\} .
$$

Let $R$ be a chamber of $H(G)$. Then an acyclic orientation of $E$ is given by

$$
\vec{E}(R)=\left\{(v, w):\{v, w\} \in E \text { and } x_{v}<x_{w} \text { for every } x \in R\right\} .
$$

Obviously, $\operatorname{Reg}(\vec{E}(R))=R$.

2.3. Hyperplane arrangements and zonotopes. The matrix $V$ in (1) defines a zonotope $Z(V)$ by

$$
Z(V)=\left\{\sum_{i=1}^{m} \alpha_{i} v_{i}:-1 \leq \alpha_{i} \leq 1\right\} .
$$

The faces of $Z(V)$ are partially ordered by inclusion. It is a well-known fact (see e.g. Zie95, Theorem 7.16]) that the partially ordered set of regions of the hyperplane arrangement $H(V)$ is anti-isomorphic to the partially ordered set of faces of $Z(V)$ : Let $R$ be a region of $H(V)$. Let $x \in R$. Then the corresponding face $\operatorname{Face}(R)$ of $Z(V)$ given by

$$
\operatorname{Face}(R)=\left\{y \in Z(V): x \cdot y=\max _{z \in Z(V)} x \cdot z\right\},
$$

does not depend on the choice of $x$. Let $F$ be a face of $Z(V)$. Let $y$ be in the relative interior of $F$. Then the corresponding region $\operatorname{Reg}(F)$ of $H(V)$ given by

$$
\operatorname{Reg}(F)=\left\{x \in \mathbb{R}^{n}: \max _{z \in Z(V)} x \cdot z=x \cdot y\right\},
$$


does not depend on the choice of $y$. Obviously, Face $(\operatorname{Reg}(F))=F$ and $F^{\prime} \subseteq F$ if and only if $\operatorname{Reg}\left(F^{\prime}\right) \supseteq \operatorname{Reg}(F)$. In particular, the chambers of $H(V)$ are in bijection with the vertices of $Z(V)$.

2.4. From lattices to zonotopes. Let $L \subseteq \mathbb{R}^{n}$ be a lattice. The support of a vector $v \in L$ is $\underline{v}=\left\{i \in\{1, \ldots, n\}: v_{i} \neq 0\right\}$. The vector $v$ is called elementary if $v \in\{-1,0,+1\}^{n} \backslash\{0\}$ and if $v$ has minimal support among all vectors in $L \backslash\{0\}$. We say that two vectors $v, w \in L$ are conformal if $v_{i} w_{i} \geq 0$ for all $i=1, \ldots, n$. The lattice $L$ is called regular if for every vector $v \in L \backslash\{0\}$ there exists an elementary vector $u \in L$ with $\underline{u} \subseteq \underline{v}$.

Lemma 1 ([Tut71, Chapter 1]).

(i) For any graph $G$ the lattice $L(G)$ is regular.

(ii) If $L$ is a regular lattice, then every $v \in L$ can be written as a sum of pairwise conformal elementary vectors.

(iii) If $L$ is a regular lattice, $v \in L$ is elementary, and $u \in L$ satisfies $\underline{u}=\underline{v}$, then there exists a factor $\alpha \in \mathbb{Z}$ such that $u=\alpha v$.

A vector $v \in L$ for which $\mathrm{V}(L) \cap\left\{x \in \mathbb{R}^{n}: x \cdot v=\frac{1}{2} v \cdot v\right\}$ is a facet of $\mathrm{V}(L)$ is called relevant. Voronoi characterizes in [Vor08, page 277] the relevant vectors of $L$ : A nonzero vector $v \in L$ is relevant if and only if $\pm v$ are the only shortest vectors in $v+2 L$.

Proposition 2. In a regular lattice, a vector is elementary if and only if it is relevant.

Proof. Let $v \in L$ be a relevant vector. By Lemma1(ii), we can write $v=\sum_{k=1}^{m} w_{k}$ as a sum of pairwise conformal elementary vectors $w_{k} \in L$. Assume that $m \geq 2$. Defining $u=v-2 w_{1}$ gives $u \neq \pm v$ and $u \cdot u=v \cdot v-4\left(v-w_{1}\right) \cdot w_{1}$. Since the vectors $w_{k}, k=1, \ldots, m$, are pairwise conformal we have $\left(v-w_{1}\right) \cdot w_{1} \geq 0$, and $\pm v$ is not the unique shortest vector in $v+2 L$. In this case $v$ cannot be a relevant vector. Hence, $m=1$ and $v$ is an elementary vector.

Let $v \in L$ be an elementary vector, and let $u \in v+2 L$ be a lattice vector with $u \neq \pm v$. We have $v-u \in 2 L \subseteq 2 \mathbb{Z}^{n}$ and $v_{i} \in\{-1,0,+1\}$, which shows $\underline{v} \subseteq \underline{u}$. The case $\underline{v} \neq \underline{u}$ immediately leads to $v \cdot v<u \cdot u$. If $\underline{v}=\underline{u}$, then by Lemma 1 (iii), there exists a factor $\alpha \in \mathbb{Z} \backslash\{-1,+1\}$ so that $u=\alpha v$, hence $v \cdot v<u \cdot u$. In both cases $\pm v$ are the only shortest vectors in $v+2 L$. Hence, $v$ is a relevant vector.

The following special case of the Farkas lemma is proved, e.g., in [Roc70, Theorem 22.6].

Lemma 2. Let $L \subseteq \mathbb{R}^{n}$ be a regular lattice. Let $x \in \mathbb{R}^{n}$ be a vector, and let $\alpha_{1}, \ldots, \alpha_{n} \in \mathbb{R} \cup\{ \pm \infty\}$. Either there exists a vector $y^{\prime} \in(\operatorname{lin} L)^{\perp}$ lying in $x+\prod_{i=1}^{n}\left[-\alpha_{i}, \alpha_{i}\right]$, or there exists a vector $y \in \operatorname{lin} L$ such that for all $z \in$ $x+\prod_{i=1}^{n}\left[-\alpha_{i}, \alpha_{i}\right]$ the inequality $y \cdot z>0$ holds. If the second condition holds, then one can choose $y$ to be an elementary vector of $L$.

Theorem 2. Let $L \subseteq \mathbb{R}^{n}$ be a regular lattice. Let $P \in \mathbb{R}^{n \times n}$ be the matrix of the orthogonal projection of $\mathbb{R}^{n}$ onto lin $L$. Then, $\mathrm{V}(L)=\frac{1}{2} Z(P)=P\left([-1 / 2,1 / 2]^{n}\right)$.

Proof. Suppose that $x \in[-1 / 2,1 / 2]^{n}$. For all $v \in \mathbb{Z}^{n} \backslash\{0\}$ the inequality $x \cdot v \leq \frac{1}{2} v \cdot v$ holds. Write $x=y+y^{\prime}$ with $y=P x \in \operatorname{lin} L$ and $y^{\prime} \in(\operatorname{lin} L)^{\perp}$. For all $v \in L \backslash\{0\}$ we have $y \cdot v=x \cdot v-y^{\prime} \cdot v \leq \frac{1}{2} v \cdot v$. Thus, $P x \in \mathrm{V}(L)$. 
Suppose now that $y \in \mathrm{V}(L)$. If there exists $x \in\left(-y+[-1 / 2,1 / 2]^{n}\right) \cap(\operatorname{lin} L)^{\perp}$, then $y+x \in[-1 / 2,1 / 2]^{n}$ and $P(y+x)=y$. Assume that such a vector does not exist. Then by Lemma 2 there is an elementary lattice vector $v \in L$ so that $v \cdot\left(-y+[-1 / 2,1 / 2]^{n}\right)>0$. This implies $v \cdot\left(-y-\frac{1}{2} v\right)>0$. Hence, $-y \notin \mathrm{V}(L)$. Since $\mathrm{V}(L)$ is centrally symmetric, this contradicts the assumption $y \in \mathrm{V}(L)$.

In Big97, Proposition 8.1] Biggs shows that for the lattice $L(G)$ the matrix $P$ can be written in the form $P=X D_{G}$ where $D_{G} \in \mathbb{R}^{V \times E}$ is the incidence matrix of $G$ and $X \in \mathbb{R}^{E \times V}$ is given by

$$
X(e, v)=\frac{\text { number of spanning trees } T \text { with } e \in T \text { and } v \in T_{e}^{+}}{\text {number of spanning trees of } G} .
$$

Furthermore, the linear map given by $X$ restricted to the image of $D_{G}$ is a bijection.

Thus, the zonotope $Z(P)$ which is the Voronoi cell of $L(G)$ equals $\frac{1}{2} X Z\left(D_{G}\right)$. Hence, there is a linear isomorphism between the faces of $\mathrm{V}(L(G))$ and those of $Z\left(D_{G}\right)$. This completes the proof of Theorem 1 .

Using a straightforward computation we get the following proposition.

Proposition 3. Using the notation in (2), the covering radius of the lattice $L(G)$ is given by

$$
\mu(L(G))^{2}=\max _{x \in[-1 / 2,1 / 2]^{E}} \sum_{e \in E}\left(\sum_{f \in E}\left(X\left(e, f^{+}\right)-X\left(e, f^{-}\right)\right) x(f)\right)^{2} .
$$

Unfortunately, we do not have a combinatorial interpretation of (3). Finding one could lead to a proof of the NP-hardness of the covering radius problem.

2.5. Lattice isomorphism problem. Using the construction $L(G)$ used in the proof of Theorem 1, we reduce the graph isomorphism problem to the lattice isomorphism problem in polynomial time. We don't know whether one can give a reverse polynomial time reduction. For the graph isomorphism problem no polynomial time algorithm is known. It is generally believed to lie in NP $\cap$ co-NP. So it is unlikely that it is NP-hard. For more information on the computational complexity of this problem, see the book KST93] of Köbler, Schöning and Tóran.

Problem 4. Lattice isomorphism problem

Input: $m, n, B, B^{\prime} \in \mathbb{Q}^{m \times n}$ matrices of rank $m$.

Output: Yes, if there is an orthogonal transformation $O$ so that $O B \mathbb{Z}^{n}=$ $B^{\prime} \mathbb{Z}^{n}$, No otherwise.

Problem 5. Graph isomorphism problem

Input: Graphs $G=\left(V, E_{G}\right), H=\left(V, E_{H}\right)$.

Output: Yes, if there is a permutation $\sigma: V \rightarrow V$ so that for all $v, w \in V$ we have $\{v, w\} \in E_{G}$ if and only if $\{\sigma(v), \sigma(w)\} \in E_{H}$, No otherwise.

Theorem 3. There is a polynomial time reduction of the graph isomorphism problem to the lattice isomorphism problem.

Proof. Let $G=\left(V, E_{G}\right)$ and $H=\left(V, E_{H}\right)$ be graphs. We modify $G$ and $H$ by adding three extra vertices to $V$ each adjacent to all vertices in $V$. We call the new graphs $G^{\prime}$ and $H^{\prime}$ which are by construction 3-connected and they are isomorphic if and only if $G$ and $H$ are isomorphic. 
It is clear that the lattices $L\left(G^{\prime}\right)$ and $L\left(H^{\prime}\right)$ defined in Subsection 2.1 are isomorphic whenever $G^{\prime}$ and $H^{\prime}$ are. For this direction it would be enough to consider the original graphs $G$ and $H$.

Now suppose that the lattices $L\left(G^{\prime}\right)$ and $L\left(H^{\prime}\right)$ are isomorphic. We apply the 2-isomorphism theorem of Whitney (actually we only use the easy subcase of 3connected graphs Oxl92, Lemma 5.3.2]): Because the graphs $G^{\prime}$ and $H^{\prime}$ are 3connected and there is a bijection between the elementary vectors preserving conformality, the graphs $G^{\prime}$ and $H^{\prime}$ are isomorphic.

\section{Algorithms}

In this section we describe an algorithm which computes all vertices of a lattice Voronoi cell. Our focus is on implementability and practical performance, using the symmetries of the lattice. In fact, the algorithm computes all full-dimensional Delone cells and the adjacencies between them up to equivalence. We give necessary definitions in Section 3.1 In Section 3.2 we describe the algorithm's main steps and in the following sections we give details about its subalgorithms. In Section 3.6. we explain how to use Gram matrices instead of lattice basis and in Section 3.7 we compare our method with existing algorithms.

3.1. Notation. From now on, we assume lattices $L \subseteq \mathbb{R}^{n}$ to have full rank $n$.

To encode the vertices of $\mathrm{V}(L)$ we use Delone cells. A point $x \in \mathbb{R}^{n}$ defines a Delone cell $D(x)$ by

$$
D(x)=\operatorname{conv}\left\{v \in L:\|x-v\|=\min _{w \in L}\|x-w\|\right\} .
$$

Denote by $S(x, r)$ the sphere with center $x$ and radius $r$. For $r=\min _{v \in L}\|x-v\|$, the sphere $S(x, r)$ is called empty, since there is no lattice point inside. In this case the polytope $D(x)$ is the convex hull of $S(x, r) \cap L$. The Delone cell of a vertex of $\mathrm{V}(L)$ is characterized among all Delone cells by the following properties: The origin is a vertex of $D(x)$ and $D(x)$ is full-dimensional.

It is well known (see e.g. [Ede01]) that the Delone cells are the projections of the faces of the infinite $(n+1)$-dimensional polyhedral set

$$
\operatorname{Lift}(L)=\operatorname{conv}\left\{\left(x,\|x\|^{2}\right): x \in L\right\} .
$$

The task of finding a vertex of a Delone cell of a point $x$, given a lattice basis of $L$, is called the closest vector problem. Generally this is an NP-hard problem DKS03; however, there are algorithms and implementations available which can solve this problem rather fast in low dimensions.

The orthogonal group $\mathrm{O}(L)$ of $L$ is the group of all orthogonal transformations $A \in \mathrm{O}\left(\mathbb{R}^{n}\right)$ fixing $L$, i.e. $A(L)=L$. The isometry group $\operatorname{Iso}(L)$ of $L$ is the group generated by $\mathrm{O}(L)$ and all lattice translations $t_{v}: \mathbb{R}^{n} \rightarrow \mathbb{R}^{n}$ with $t_{v}(x)=x+v$ for $v \in L$.

We say that two vertices $x$ and $x^{\prime}$ of $\mathrm{V}(L)$ are equivalent if there is an $A \in \mathrm{O}(L)$ so that $A(x)=x^{\prime}$. Correspondingly, we say that two Delone cells $D(x)$ and $D\left(x^{\prime}\right)$ are equivalent if there is an $A \in \operatorname{Iso}(L)$ so that $A(D(x))=D\left(x^{\prime}\right)$.

3.2. Main algorithm. Our algorithm finds a complete list of inequivalent fulldimensional Delone cells of $L$ with respect to $\operatorname{Iso}(L)$. The enumeration process is a graph traversal algorithm of the graph of equivalence classes of full-dimensional 
Delone cells of $L$. Two equivalence classes are adjacent whenever there is a facet between two of its representatives. Note that this graph can have loops and multiple edges.

For the graph traversal algorithm below one needs four subalgorithms, which we explain in the following sections.

Input: $n, B \in \mathbb{Q}^{n \times n}$ matrix of rank $n$.

Output: Set $\mathcal{M}$ of all inequivalent full-dimensional Delone cells of the lattice

$B \mathbb{Z}^{n}$ with respect to the group $\operatorname{Iso}\left(B \mathbb{Z}^{n}\right)$.

$x \leftarrow$ an initial vertex of $\mathrm{V}\left(B \mathbb{Z}^{n}\right)$.

(Section 3.3)

$T \leftarrow\{D(x)\}$.

$\mathcal{M} \leftarrow \emptyset$.

while there is a $D \in T$ do

$\mathcal{M} \leftarrow \mathcal{M} \cup\{D\}$.

$T \leftarrow T \backslash\{D\}$.

$\mathcal{F} \leftarrow$ facets of $D$.

(Section 3.4)

for $F \in \mathcal{F}$ do

$D^{\prime} \leftarrow$ full-dimensional Delone cell with $F=D \cap D^{\prime}$.

if $D^{\prime}$ is not equivalent to a Delone cell in $\mathcal{M} \cup T$ then

(Section 3.4)

$T \leftarrow T \cup\left\{D^{\prime}\right\}$.

end if

end for

end while

Two full-dimensional Delone cells $D(x)$ and $v+D(x)$, both containing the origin, are equivalent under $\mathrm{O}(L)$ if and only if 0 and $-v$ are equivalent under the stabilizer group of $D(x)$ in Iso $(L)$. As a consequence, we can compute the vertices of $\mathrm{V}(L)$ under $\mathrm{O}(L)$ in the following way: For every orbit of full-dimensional Delone cells given by a representative $D(x)$, we compute the orbits of vertices of $D(x)$ under the stabilizer group and get the corresponding orbits of vertices of $\mathrm{V}(L)$ under $\mathrm{O}(L)$.

3.3. Finding an initial vertex. Now we explain a method for computing an initial vertex of the Voronoi cell of a lattice, i.e. a full-dimensional Delone cell containing the origin. The method we propose is a so-called cutting-plane algorithm, which is a well-known technique in combinatorial optimization.

Let us describe the geometric idea. We start with an outer approximation of the Voronoi cell given by linear inequalities. The first outer approximation is the polytope defined by the inequalities $\pm b_{i} \cdot x \leq \frac{1}{2} b_{i} \cdot b_{i}$ for given lattice basis vectors $b_{1}, \ldots, b_{n}$. Then we find a vertex $x$ of the approximation by linear programming (see e.g. [Sch86]). Deciding whether the vertex $x$ belongs to the Voronoi cell $\mathrm{V}(L)$ can be done as follows: Compute the vertices of the Delone cell $D(x)$. If the origin is a vertex of $D(x)$, then $x$ is a vertex of $\mathrm{V}(L)$. Otherwise $x$ is not contained in $\mathrm{V}(L)$, and for all vertices $v$ of $D(x)$ we have the strict inequality $\|x-v\|<\|v\|$. So the new linear inequalities $v \cdot x \leq \frac{1}{2} v \cdot v$ together with the old ones provide a tighter outer approximation of the Voronoi cell. Since we started with a compact outer approximation, finitely many iterations of these steps suffice to find a vertex of the Voronoi cell.

One advantage of this method is that the computation of all facets of the Voronoi cell is not required, i.e. we do not use Voronoi's characterization (see Section 2.4) 


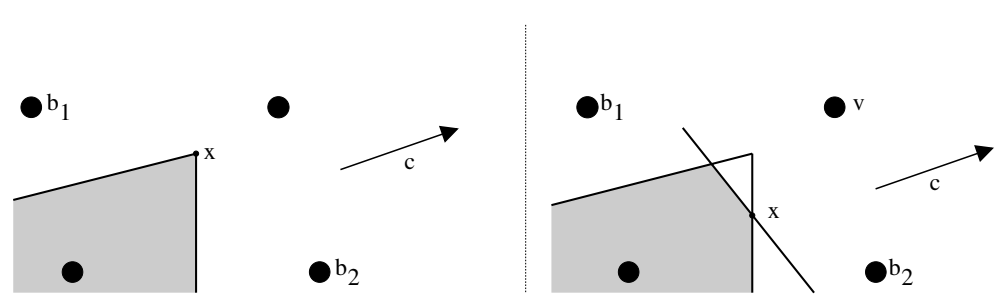

Figure 1. Finding an initial vertex of $\mathrm{V}(L)$

of facet defining vectors, which involves solving exponentially many closest vector problems. Figure 1 illustrates this algorithm.

Input: $n, B=\left(b_{1}, \ldots, b_{n}\right) \in \mathbb{Q}^{n \times n}$ matrix of rank $n$.

Output: vertex $x$ of $\mathrm{V}\left(B \mathbb{Z}^{n}\right)$.

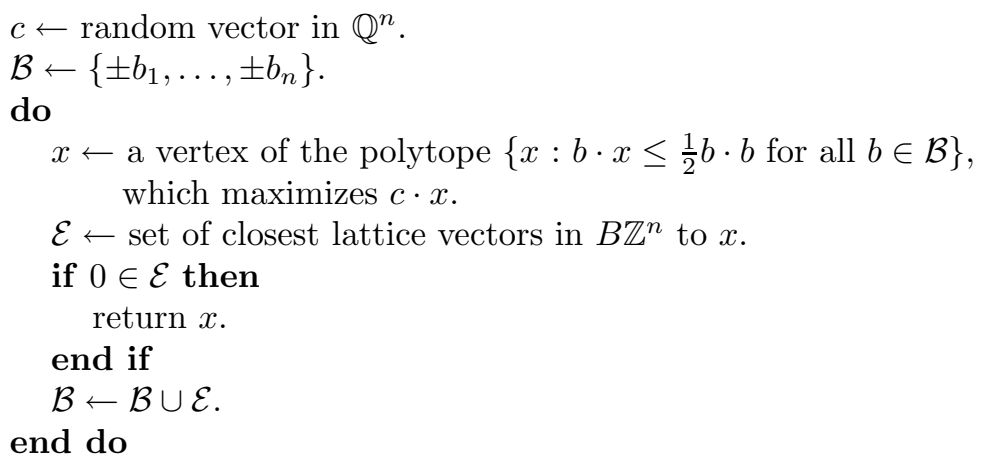

3.4. Computing facets of, and finding adjacent Delone cells. We want to determine the facets of a full-dimensional Delone cell, which is given by its vertex set. This representation conversion problem can be solved by many different methods. For details and implementations we refer to cdd [Fuk95], Irs [Avi93, pd Mar97. and porta [CL97.

In order to exploit the symmetries we use the adjacency decomposition method (see CR96, BDS07, DSV07]). It allows us to compute a complete list of inequivalent facet representatives: We compute an initial facet by linear programming and insert it into the list of orbit representatives of facets. From any such orbit, we compute the list of facets adjacent to a representative and insert it, if necessary, into the list of representatives until all orbits have been treated. Computing adjacent facets is itself a representation conversion problem in one dimension lower. So this method can be applied recursively (see BDS07, DSV07]). Note that our main algorithm is itself an adjacency decomposition method.

After the computation of facets, we can compute adjacent full-dimensional Delone cells: We take an initial vertex $v$ and thus get a tentative empty sphere. If the sphere is not empty, then we find another vertex $v$ and iterate until the sphere is indeed empty. Figure 2 illustrates this algorithm. 

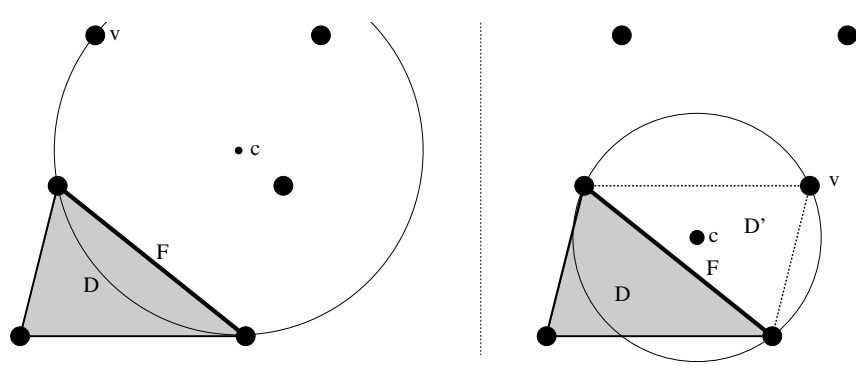

Figure 2. Finding $D^{\prime}$, the full-dimensional Delone cell adjacent to $D$ at $F$

Input: $n, B \in \mathbb{Q}^{n \times n}$ matrix of rank $n$, a full-dimensional Delone cell $D$ and a facet $F$ of $D$.

Output: Vertex set $\mathcal{V}^{\prime}$ of a full-dimensional Delone cell $D^{\prime}$ with $D \cap D^{\prime}=F$.

$\phi \leftarrow$ affine function on $\mathbb{R}^{n}$ with $F=\{x \in D: \phi(x)=0\}$ and $\phi(x)>0$

on $D-F$.

$\mathcal{V}_{F} \leftarrow$ vertices of $D$ belonging to $F$.

$v \leftarrow$ a point of $B \mathbb{Z}^{n}$ with $\phi(v)<0$.

do

$S(c, r) \leftarrow$ sphere around $\mathcal{V}_{F} \cup\{v\}$.

$\mathcal{V}^{\prime} \leftarrow$ closest vectors in $B \mathbb{Z}^{n}$ to $c$.

if $\left\|v^{\prime}-c\right\|=r$ for a $v^{\prime} \in \mathcal{V}^{\prime}$ then

return $\mathcal{V}^{\prime}$

end if

$v \leftarrow$ one element of $\mathcal{V}^{\prime}$.

end do

One way to speed up the convergence of this algorithm in practice is to heuristically choose an initial vector $v$ with a sphere $S(c, r)$ of small radius.

3.5. Checking equivalence. We have to test equivalence and compute stabilizers under the group Iso $(L)$ of Delone cells of different dimensions. Below we propose three different methods for this.

We can encode a Delone cell $D$ by the center $c(D)$ of the empty sphere around it or by the vertex barycenter $g(D)=\frac{1}{|\operatorname{vert} D|} \sum_{v \in \operatorname{vert} D} v$ of its vertex set vert $D$. Both $c(D)$ and $g(D)$ are invariant under the stabilizer of $D$. Any two full-dimensional Delone cells $D, D^{\prime}$ are equal if and only if $c(D)=c\left(D^{\prime}\right)$. However, it is possible if $n \geq 3$ that $c(D)$ lies outside or on the boundary of $D$. If $c(D)$ lies on the boundary of $D$, then a facet containing $c(D)$, which is itself a Delone cell, has the same center as $D$. Hence, the sphere centers can be used to distinguish full-dimensional Delone cells, but they do not distinguish Delone cells. Therefore, we use the vertex barycenter.

In the first method we consider the classes of the vertex barycenters $g(D)$ and $g\left(D^{\prime}\right)$ in the quotient $\mathbb{R}^{n} / L$ and check their equivalence under the finite group Iso $(L) / L \simeq \mathrm{O}(L)$. The generic methods underlying isomorphism and stabilizer computations generate the full orbit of $g(D)$ under $\operatorname{Iso}(L) / L$. This is typically memory intensive. In some cases we can use a method from computational group theory, which we now explain in an example. Suppose $g(D)$ is expressed in a 
basis $\left(b_{1}, \ldots, b_{n}\right)$ of $L$ as $\left(\frac{\alpha_{1}}{2 \cdot 3}, \ldots, \frac{\alpha_{n}}{2 \cdot 3}\right)$ with $0 \leq \alpha_{i} \leq 5$ and we want to compute its stabilizer under the group $\operatorname{Iso}(L) / L$. The vector $2 g(D)$ reduced modulo $L$ is expressed as $\left(\frac{\tilde{\alpha}_{1}}{3}, \ldots, \frac{\tilde{\alpha}_{n}}{3}\right)$ with $0 \leq \tilde{\alpha}_{i} \leq 2$. We first compute the stabilizer $H$ of the vector $2 g(D)$ under the action of $\operatorname{Iso}(L) / L$. The stabilizer of $g(D)$ under Iso $(L) / L$ is equal to the stabilizer of $g(D)$ under $H$. This method generalizes to more than two prime factors and it is more memory efficient because the generated orbits are smaller.

The second method uses finite metric spaces of the vertex set of full-dimensional Delone cells obtained from the metric $\left\|v-v^{\prime}\right\|^{2}$ [DL97, Chapter 14]. A finite metric space defines an edge-weighted graph. Testing if two edge weighted graphs are isomorphic can be reduced to testing if two vertex-weighted graphs are isomorphic (see [McK06, p. 25]). In practice, the program nauty [McK06] can solve the isomorphism problem if the number of vertices of $D$ and $D^{\prime}$ is not too large. If the metric spaces are not isomorphic, then $D$ and $D^{\prime}$ are not equivalent under $\operatorname{Iso}(L)$. If they are isomorphic, then every graph isomorphism corresponds to a linear isometry between $D$ and $D^{\prime}$ [BDS07, DSV07. For each of those isomorphisms, we check if it belongs to Iso $(L)$. This method is useful when the isometry group of $D$ is small.

For the third method, we use laminations over the $n$-dimensional lattice $L$. Let $D$ be a Delone cell of $L$ with vertex barycenter $g$. One defines an $(n+1)$-dimensional lattice $L(g)$ by embedding $L \subseteq \mathbb{R}^{n}$ into $\mathbb{R}^{n+1}$ and adding layers to it

$$
L(g)=\{\alpha v+h: h \in L, \alpha \in \mathbb{Z}\} \subseteq \mathbb{R}^{n+1},
$$

where $v \in \mathbb{R}^{n+1}$ is chosen so that $v+g$ is orthogonal to the space spanned by $L$ and normalized so that $\|v+g\|=1$. A variant of this construction is used, for example, to build the laminated lattices; see [CS99, Chapter 6]. If $\phi$ is an element of $\mathrm{O}(L(g))$ preserving every layer of the lamination, then it maps the vector $v$ to some vector $w=v+h$ with $h \in L$. The function $x \mapsto \phi(x)+h$ preserves the Delone cell and every element preserving the layers is obtained in this way. In practice, we can use the program AUTO (see [PP97]) of the package CARAT (see OPS98) for computing this automorphism group. The isomorphism problem is treated similarly using the program ISOM.

3.6. Working with Gram matrices and periodic structures. In many cases, it is more convenient to work with the Gram matrix $B^{T} B$ instead of the lattice basis $B$ (see [CS99, Chapter 2.2]). For instance, when $B$ is irrational but $B^{T} B$ is rational. Note that our algorithms can be reformulated in terms of Gram matrices. Note also that all of our algorithms can be modified to deal with periodic point sets, that is for finite unions of lattice translates. Our implementation is available from [Dut08].

3.7. Comparison. In VB96 Viterbo and Biglier describe another algorithm for computing the Voronoi cell of a lattice, called the diamond cutting algorithm. As in our approach they start with a parallelepiped $P$ defined by the basis vectors. Then they determine all lattice vectors which lie in a sphere containing $2 P$. This set contains all facet defining lattice vectors of $\mathrm{V}(L)$. Successively they add cutting planes obtained from these vectors and update the complete face lattice of the tentative Voronoi cell. They terminate when its volume coincides with $\operatorname{det} L$. Their implementation uses floating point arithmetic. 
In comparison, our approach has the following advantages: We use the presence of symmetry in an efficient way. We do not need to compute a huge initial list of potential facet defining lattice vectors. Our algorithm does not need to compute the face lattice, not even for computing the quantizer constant as explained below. Our implementation uses rational arithmetic only.

\section{Computing Quantizer COnstants}

Recall from the introduction that the quantizer constant of a lattice $L$ is the integral

$$
G(L)=(\operatorname{det} L)^{-(1+2 / n)} \int_{\mathrm{V}(L)}\|x\|^{2} d x .
$$

A standard method for computing the integral $G(L)$ is to decompose $\mathrm{V}(L)$ into simplices. Suppose that $S$ is a simplex with vertices $v_{1}, \ldots, v_{n+1}$ in $\mathbb{R}^{n}$. Then (see CS99, Chapter 21, Theorem 2]) the following holds:

$$
\int_{S}\|x\|^{2} d x=\frac{\operatorname{vol} S}{(n+1)(n+2)}\left(\left\|\sum_{i=1}^{n+1} v_{i}\right\|^{2}+\sum_{i=1}^{n+1}\left\|v_{i}\right\|^{2}\right) .
$$

Thus $G(L)$ can be obtained by summing the integrals of all simplices in a decomposition of $\mathrm{V}(L)$. Several practical methods for decomposing a polytope into simplices are discussed in BEF98. In our implementation, we use the triangulation obtained by the program lrs. However, this method as well as the other methods explained in BEF98 are sometimes impractical and they do not use symmetries.

In order to get a group invariant decomposition, we can use the barycentric subdivision of $P$. That is, given any flag $F_{0} \subset F_{1} \subset \cdots \subset F_{n}=P$ of faces of $P$, we associate the simplex with vertex set $g_{0}, g_{1}, \ldots, g_{n}$ where $g_{i}$ is the vertex barycenter of $F_{i}$. Note that, in general, there is a difference between the barycenter $\frac{1}{\operatorname{vol} P} \int_{P} x d x$ of a polytope $P$ and its vertex barycenter $\frac{1}{|\operatorname{vert} P|} \sum_{v \in \text { vert } P} v$. The group acts on the barycentric subdivision and the stabilizer of each simplex is trivial. In practice, the number of orbits of flags can be too large.

We propose a hybrid approach, which combines the benefits of both methods. Let $\mathcal{F}$ be the facet set of an $n$-dimensional polytope $P$. We can assume, without loss of generality, that $P$ has the origin as its vertex barycenter. We then have

$$
\int_{P}\|x\|^{2} d x=\sum_{F \in \mathcal{F}} \int_{\operatorname{conv}(F, 0)}\|x\|^{2} d x .
$$

To compute this sum, it is sufficient to compute the integrals only for orbit representatives of facets. Let $F$ be a facet of $P$ and $p_{F}$ a point in the affine space spanned by $F$. Then we can transform the integral over the cone $\operatorname{conv}(F, 0)$ in the following way:

$$
\begin{array}{r}
\int_{\operatorname{conv}(F, 0)}\|x\|^{2} d x=\frac{1}{n+2}\left(\int_{F}\left\|y-p_{F}\right\|^{2} d y\right. \\
\left.+2 \int_{F}\left(y-p_{F}\right) \cdot p_{F} d y+\operatorname{vol} F\left\|p_{F}\right\|^{2}\right) .
\end{array}
$$

If $p_{F}$ is the orthogonal projection of the origin 0 onto $F$, then the second summand vanishes. This point may not be invariant under the automorphism group of the facet $F$, but the vertex barycenter is. If we use the vertex barycenter, we also 
have to compute the barycenter of the polytope $F$ as well as the volume and the square integral. In order to use symmetries coming from nonorthogonal linear transformations of $P$, we use the matrix-valued integral

$$
I_{0,1,2}(P)=\int_{P}\left(\begin{array}{c}
1 \\
x
\end{array}\right)\left(1, x^{t}\right) d x .
$$

This integral splits according to

$$
I_{0,1,2}(P)=\left(\begin{array}{cc}
I_{0}(P) & I_{1}(P)^{t} \\
I_{1}(P) & I_{2}(P)
\end{array}\right)
$$

where

$$
I_{0}(P)=\int_{P} d x=\operatorname{vol} P, \quad I_{1}(P)=\int_{P} x d x, \quad I_{2}(P)=\int_{P} x x^{t} d x .
$$

Let $G$ be a group of automorphisms of $P$. If $g \in G$ acts on $\mathbb{R}^{n}$ as $x \mapsto A x+v$, then we define $H(g)=\left(\begin{array}{ll}1 & 0 \\ v & A\end{array}\right)$ as the corresponding $(n+1) \times(n+1)$ matrix acting on homogeneous coordinates. Let $O_{1}, \ldots, O_{r}$ be the $G$-orbits of facets of $P$, with representatives $F_{1}, \ldots, F_{r}$. Then the integral $I_{0,1,2}(P)$ simplifies to

$$
I_{0,1,2}(P)=\sum_{i=1}^{r}\left|O_{i}\right|\left(\frac{1}{|G|} \sum_{g \in G} H(g) I_{0,1,2}\left(\operatorname{conv}\left(F_{i}, 0\right)\right) H(g)^{t}\right) .
$$

Assume that $I_{0,1,2}\left(\operatorname{conv}\left(F_{i}, 0\right)\right)$ is already computed. To compute the sum in the parenthesis, we first incrementally compute a basis of the affine hull of the orbit $\left\{H(g) I_{0,1,2}\left(\operatorname{conv}\left(F_{i}, 0\right)\right) H(g)^{t}: g \in G\right\}$. The only $G$-invariant element of the affine hull is the sum we want to compute.

We now want to compute $I_{0,1,2}(\operatorname{conv}(F, 0))$ in terms of lower dimensional integrals. The integral depends on the chosen basis. If $f$ is an affine transformation of $\mathbb{R}^{n}$, then the change of variables formula for integrals gives

$$
H(f) I_{0,1,2}(f P) H(f)^{t}|\operatorname{det} H(f)|=I_{0,1,2}(P),
$$

for any $n$-dimensional polytope $P$ in $\mathbb{R}^{n}$. This allows us to compute $I_{0,1,2}(P)$ for another basis. So, we can choose a coordinate system such that

$$
F=\left\{\left(\begin{array}{l}
1 \\
x
\end{array}\right): x \in F^{\prime}\right\} \subset \mathbb{R}^{n}
$$

where $F^{\prime} \subset \mathbb{R}^{n-1}$ is an $(n-1)$-dimensional polytope. We then have the following formulas:

$$
\begin{aligned}
I_{0}(\operatorname{conv}(F, 0))= & \frac{1}{n} I_{0}\left(F^{\prime}\right), \quad I_{1}(\operatorname{conv}(F, 0))=\frac{1}{n+1}\left(\begin{array}{c}
I_{0}\left(F^{\prime}\right) \\
I_{1}\left(F^{\prime}\right)
\end{array}\right), \\
& I_{2}(\operatorname{conv}(F, 0))=\frac{1}{n+2} I_{0,1,2}\left(F^{\prime}\right) .
\end{aligned}
$$

For computing $I_{0,1,2}\left(F^{\prime}\right)$, we have two options: Either we use the first method of this section, which involves computing a triangulation or we apply the above method recursively. The decision is made heuristically, depending on the size of the automorphism group of $F$ and its number of vertices. In order to reduce the size of the computation, one can store intermediate results.

Those methods are general and apply to any polytope and any polynomial function, which we want to integrate over $P$. Note that a similar method of using the standard formula (4) has been used for computing the volume in BEF98, under the name of Lasserre's method ([Las98] $)$, albeit in a nongroup setting. 


\section{RESULTS}

In this section, we collect results from our implementation of the algorithms explained in Sections 3 and 4 . We obtain previously unknown exact covering densities and quantizing constants of several prominent lattices and their duals. Recall that the dual $L^{*}$ of a lattice $L \subset \mathbb{R}^{n}$ is defined by

$$
L^{*}=\left\{x \in \mathbb{R}^{n}: y \cdot x \in \mathbb{Z} \quad \text { for all } y \in L\right\} .
$$

The covering density of an $n$-dimensional lattice $L$ is

$$
\frac{\mu(L)^{n}}{\operatorname{det} L} \operatorname{vol} B_{n},
$$

where $B_{n}$ is the unit ball in $\mathbb{R}^{n}$. Other computations of Voronoi cells of lattices can be found in [CS91, [EMS03, Chapter 5] and [MP95]. All computations are done in exact rational arithmetic. In the tables the covering densities are given in floating point; the exact expressions would be too large.

5.1. Coxeter lattices. The root lattice $\mathrm{A}_{n}$ is defined by

$$
\mathrm{A}_{n}=\left\{x \in \mathbb{Z}^{n+1}: \sum_{i=1}^{n+1} x_{i}=0\right\} .
$$

If $r$ divides $n+1$, the Coxeter lattice $\mathrm{A}_{n}^{r}$ (see [Cox51]) is defined by translates of $\mathrm{A}_{n}$ :

$$
\mathrm{A}_{n}^{r}=\mathrm{A}_{n} \cup\left(v_{n}^{r}+\mathrm{A}_{n}\right) \cup \ldots \cup\left((r-1) v_{n}^{r}+\mathrm{A}_{n}\right),
$$

where $v_{n}^{r}=\frac{1}{n+1} \sum_{i=2}^{n+1}\left(e_{i}-e_{1}\right)$. The dual lattice of $\mathrm{A}_{n}^{r}$ is $\mathrm{A}_{n}^{n+1 / r}$.

The Delone decomposition of the lattice $\mathrm{A}_{n}^{r}$ has been studied in Anz02, Anz06, Bar94 up to dimension $n=15$, hoping to obtain lattices with low covering density. One pleasant fact is that the symmetry group of $\mathrm{A}_{n}^{r}$ contains the group $\operatorname{Sym}(n+$ $1) \times \mathbb{Z}_{2}$. The latter can be represented as a permutation group acting on $n+3$ points, which drastically simplifies isomorphism computations.

In Table 1 we list the obtained results. Note that the lattices $A_{17}^{6}, A_{19}^{10}, A_{20}^{7}$ and $A_{21}^{11}$ turn out to give new record sphere coverings. Up to dimension 8 all of those lattices are well known and their Voronoi cells can be obtained by standard computer algebra software. Our list is complete up to dimension 21. For the missing cases we could not finish the computation.

5.2. Laminated lattices. The laminated lattices, which are defined in CS99, Chapter 6], give the best known lattice sphere packings in many dimensions. The Delone subdivision is known up to dimension 8 and in dimension 24 for all laminated lattices and their duals CS99. Chapters 21, 23, 25]. In dimension 16, the covering density of $\Lambda_{16}$ is known [CS99, Chapter 6].

In Table 2 we list the obtained results, which are complete up to dimension 17.

5.3. Shorter Leech lattice. The 4600 shortest vectors of $\Lambda_{23}^{*}$ define a sublattice of index 2, called the shorter Leech lattice $O_{23}$ ([CS99, pp. 179, 420, 441]). The Delone decomposition (see Table 3) is remarkable in many respects: There are only 5 orbits and the first one has the full symmetry group of the lattice. It turns out that $\Lambda_{23}^{*}=O_{23} \cup\left(v+O_{23}\right)$ where $v$ is the center of a centrally symmetric Delone cell lying in the first orbit. The covering density of $O_{23}$ is 15218.062669 .

5.4. Cut lattices. The cut polytope $\mathrm{CUT}_{n}$ is a famous polytope appearing in combinatorial optimization (see [DL97]). It has $2^{n-1}$ vertices and is of dimension 
$\frac{n(n-1)}{2}$. The lattice generated by its vertices is called cut lattice and is denoted by $L\left(\mathrm{CUT}_{n}\right)$ (see [DG95]). The polytope $\mathrm{CUT}_{n}$ is one of its full-dimensional Delone cells. We list our results in Table 4.

5.5. Quantizer constants. In Table 5 we collect some new exact quantizer constants.

According to AE98, the lattice $\mathrm{D}_{10}^{+}$is conjectured to be the optimal lattice quantizer. Conway and Sloane approximated $G\left(\mathrm{~K}_{12}\right)$ ([CS99, Table 2.3]) using Monte-Carlo integration; our exact computation fits into their bounds.

TABLE 1. Number of orbits of full-dimensional Delone cells and covering density for some Coxeter lattices

\begin{tabular}{c|c|c|c|c|c} 
lattice & \# orbits & covering density & lattice & \# orbits & covering density \\
\hline $\mathrm{A}_{9}^{2}$ & 6 & 18.543333 & $\mathrm{~A}_{9}^{5}$ & 5 & 4.340184 \\
\hline $\mathrm{A}_{11}^{2}$ & 6 & 94.090996 & $\mathrm{~A}_{11}^{3}$ & 11 & 27.089662 \\
$\mathrm{~A}_{11}^{4}$ & 16 & 5.598337 & $\mathrm{~A}_{11}^{6}$ & 4 & 7.618558 \\
\hline $\mathrm{A}_{13}^{2}$ & 10 & 134.623484 & $\mathrm{~A}_{13}^{7}$ & 10 & 7.864060 \\
\hline $\mathrm{A}_{14}^{3}$ & 17 & 32.313517 & $\mathrm{~A}_{14}^{5}$ & 31 & 9.006610 \\
\hline $\mathrm{A}_{15}^{2}$ & 10 & 722.452642 & $\mathrm{~A}_{15}^{4}$ & 19 & 25.363859 \\
$\mathrm{~A}_{15}^{8}$ & 10 & 11.601626 & & & \\
\hline $\mathrm{A}_{17}^{2}$ & 15 & 1068.513081 & $\mathrm{~A}_{17}^{3}$ & 26 & 240.511580 \\
$\mathrm{~A}_{17}^{6}$ & 73 & 12.357468 & $\mathrm{~A}_{17}^{9}$ & 24 & 17.231927 \\
\hline $\mathrm{A}_{19}^{2}$ & 15 & 5921.056764 & $\mathrm{~A}_{19}^{4}$ & 58 & 40.445924 \\
$\mathrm{~A}_{19}^{5}$ & 80 & 25.609662 & $\mathrm{~A}_{19}^{10}$ & 80 & 21.229200 \\
\hline $\mathrm{A}_{20}^{3}$ & 40 & 307.209487 & $\mathrm{~A}_{20}^{7}$ & 187 & 20.366828 \\
\hline $\mathrm{A}_{21}^{2}$ & 21 & 8937.567486 & $\mathrm{~A}_{21}^{11}$ & 64 & 27.773140 \\
\hline $\mathrm{A}_{23}^{3}$ & 55 & 2405.032746 & $\mathrm{~A}_{23}^{4}$ & 85 & 205.561225 \\
$\mathrm{~A}_{23}^{6}$ & 187 & 79.575330 & $\mathrm{~A}_{23}^{8}$ & 495 & 31.858162 \\
$\mathrm{~A}_{23}^{12}$ & 100 & 43.231587 & & & \\
\hline $\mathrm{A}_{24}^{5}$ & 144 & 115.011591 & & & \\
\hline $\mathrm{A}_{25}^{13}$ & 210 & 54.472182 & & & \\
\hline $\mathrm{A}_{26}^{3}$ & 75 & 3184.1387034 & $\mathrm{~A}_{26}^{9}$ & 1231 & 50.937168 \\
\hline $\mathrm{A}_{27}^{4}$ & 156 & 350.137031 & $\mathrm{~A}_{27}^{7}$ & 650 & 81.869181 \\
\hline $\mathrm{A}_{27}^{14}$ & 338 & 76.909712 & & & \\
\hline $\mathrm{A}_{29}^{3}$ & 102 & 25664.644103 & $\mathrm{~A}_{29}^{5}$ & 347 & 202.040331 \\
$\mathrm{~A}_{29}^{6}$ & 711 & 154.329831 & $\mathrm{~A}_{29}^{10}$ & 3581 & 84.324725 \\
$\mathrm{~A}_{29}^{15}$ & 678 & 114.084219 & & & \\
\hline $\mathrm{A}_{31}^{16}$ & 1225 & 33.934941 & & & \\
\hline & & & & & \\
\hline
\end{tabular}


TABle 2. Number of orbits of full-dimensional Delone cells and covering density for some laminated lattices and their duals

\begin{tabular}{c|c|c|c|c|c} 
lattice & \# orbits & covering density & lattice & \# orbits & covering density \\
\hline$\Lambda_{9}$ & 5 & 9.003527 & $\Lambda_{9}^{*}$ & 9 & 9.003527 \\
\hline$\Lambda_{10}$ & 7 & 12.408839 & $\Lambda_{10}^{*}$ & 21 & 9.306629 \\
\hline$\Lambda_{11}^{\text {max }}$ & 11 & 24.781167 & $\Lambda_{11}^{\text {max* }}$ & 18 & 19.243468 \\
$\Lambda_{11}^{\text {min }}$ & 18 & 24.781167 & $\Lambda_{11}^{\text {min* }}$ & 153 & 8.170432 \\
\hline$\Lambda_{12}^{\text {max }}$ & 5 & 30.418954 & $\Lambda_{12}^{\text {max* }}$ & 8 & 42.728408 \\
$\Lambda_{12}^{\text {mid }}$ & 23 & 30.418954 & $\Lambda_{12}^{\text {mid } *}$ & 52 & 19.176309 \\
$\Lambda_{12}^{\text {min }}$ & 13 & 30.418954 & $\Lambda_{12}^{\text {min* }}$ & 78 & 12.292973 \\
\hline$\Lambda_{13}^{\text {max }}$ & 18 & 60.455139 & $\Lambda_{13}^{\text {max* }}$ & 57 & 43.214494 \\
$\Lambda_{13}^{\text {mid }}$ & 46 & 35.931846 & $\Lambda_{13}^{\text {mid }}$ & 125 & 19.155991 \\
$\Lambda_{13}^{\text {min }}$ & 129 & 60.455139 & $\Lambda_{13}^{\text {min* }}$ & 5683 & 13.724864 \\
\hline$\Lambda_{14}$ & 65 & 98.875610 & $\Lambda_{14}^{*}$ & 1392 & 34.721750 \\
\hline$\Lambda_{15}$ & 27 & 202.910873 & $\Lambda_{15}^{*}$ & 108 & 25.642067 \\
\hline$\Lambda_{16}$ & 4 & 96.500266 & $\Lambda_{16}^{*}$ & 4 & 96.500266 \\
\hline$\Lambda_{17}$ & 28 & 197.719499 & $\Lambda_{17}^{*}$ & 720 & 100.173101 \\
\hline$\Lambda_{18}$ & 239 & 301.192334 & & & \\
\hline$\Lambda_{23}$ & 709 & 7609.03133 & & &
\end{tabular}

TABle 3. Orbits of full-dimensional Delone cells of $O_{23}$

\begin{tabular}{c|c} 
number of vertices & size of stabilizer group \\
\hline 94208 & 84610842624000 \\
32 & 1344 \\
24 & 10200960 \\
24 & 1320 \\
24 & 1320
\end{tabular}

TABLE 4. Dimensions, number of orbits of full-dimensional Delone cells and covering density of some cut lattices

\begin{tabular}{c|c|c|c} 
lattice & dimension & \# orbits & covering density \\
\hline$L\left(\mathrm{CUT}_{3}\right)$ & 3 & 2 & 2.09439 \\
$L\left(\mathrm{CUT}_{4}\right)$ & 6 & 4 & 5.16771 \\
$L\left(\mathrm{CUT}_{5}\right)$ & 10 & 12 & 40.80262 \\
$L\left(\mathrm{CUT}_{6}\right)$ & 15 & 112 & 255.4255
\end{tabular}


TABLE 5. Quantizer constants of some lattices

\begin{tabular}{c|c} 
lattice & quantizer constant \\
\hline$\Lambda_{9}$ & $\frac{151301}{2099520} \approx 0.07206$ \\
$\Lambda_{9}^{*}$ & $\frac{1371514291}{19110297600} \approx 0.07176$ \\
$\mathrm{~A}_{9}^{2}$ & $\frac{2120743}{\sqrt[9]{5.2^{8}} 13271040} \approx 0.072166$ \\
$\mathrm{~A}_{9}^{5}$ & $\frac{8651427563}{\sqrt[9]{2.5^{8}} 26578125000} \approx 0.072079$ \\
$\mathrm{D}_{10}^{+}$ & $\frac{4568341}{64512000} \approx 0.07081$ \\
$\mathrm{~A}_{11}^{2}$ & $\frac{452059}{\sqrt[11]{3} 5702400} \approx 0.07174$ \\
$\mathrm{~A}_{11}^{3}$ & $\frac{287544281699}{\sqrt[11]{4.3^{10}} 1325839006800} \approx 0.070426$ \\
$\mathrm{~A}_{11}^{4}$ & $\frac{6387657954959}{\sqrt[11]{3.2^{9}} 46506442752000} \approx 0.070494$ \\
$\mathrm{D}_{12}^{+}$ & $\frac{29183629}{412776000} \approx 0.070700$ \\
$\mathrm{~K}_{12}$ & $\frac{797361941}{\sqrt{3} 6567561000} \approx 0.070095$
\end{tabular}

\section{ACKNOWLEDGEMENTS}

We thank David Avis, Andreas Enge, Alexander Hulpke, Gabriele Nebe, Warren Smith, Bernd Souvignier and the anonymous referees for helpful discussions and suggestions.

\section{REFERENCES}

[AE98] E. Agrell, T. Eriksson, Optimization of lattices for quantization, IEEE Trans. Inform. Theory 44 (1998) 1814-1828. MR1664114 (99h:94003)

[Anz02] M. M. Anzin, On the density of a lattice covering for $n=11$ and $n=14$, Russian Math. Surveys 57 (2002) 407-409, translation from Uspekhi Mat. Nauk 57, (2002) 187-188. MR:1918199(2003m:11098)

[Anz06] M. M. Anzin, On the density of the lattice covering for $n=13$ and $n=15$, Math. Notes 79 (2006) 721-725, translation from Mat. Zametki 79 (2006) 781-784. MR 2249136 (2007f:11075)

[Avi93] D. Avis, A C-implementation of the reverse search vertex enumeration algorithm, School of Computer Science, McGill University, Montreal, Canada 1993, http://www-cgrl.cs.mcgill.ca/ avis/C/lrs.html. MR1330479

[Bar94] E. P. Baranovskii, The perfect lattices $\Gamma\left(\mathfrak{A}^{n}\right)$, and the covering density of $\Gamma\left(\mathfrak{A}^{9}\right)$, European J. Combin. 15 (1994), 317-323. MR1279070 (95i:11069)

[Big97] N. Biggs, Algebraic potential theory on graphs, Bull. London Math. Soc. 29 (1997), 641-682. MR 1468054 (98m:05120)

[BDS07] D. Bremner, M. Dutour Sikirić, A. Schürmann, Polyhedral representation conversion up to symmetries, preprint, September 2007, arXiv:math/0702239v2 [math.MG].

[BEF98] B. Büeler, A. Enge, K. Fukuda, Exact volume computation for polytopes: a practical study, pages 131-154 in Polytopes - combinatorics and computation (Oberwolfach, 1997), Birkhäuser, 2000. MR1785296 (2001e:52025)

[CL97] T. Christof, A. Löbel, PORTA: Polyhedron representation transformation algorithm (ver 1.3.1), 1997, http://www.zib.de/Optimization/Software/Porta/.

[CR96] T. Christof, G. Reinelt, Combinatorial optimization and small polytopes, Top 4 (1996) 1-64. MR1404262 (97g:90107)

[CS91] J. H. Conway, N. J. A. Sloane, The cell structures of certain lattices, pages 71-107 in Miscellanea mathematica, Springer, 1991. MR1131118(92i:52019) 
[CS99] J. H. Conway, N. J. A. Sloane, Sphere packings, lattices and groups (third edition), Springer, 1999. MR1662447 (2000b:11077)

[Cox51] H. S. M. Coxeter, Extreme forms, Canadian J. Math. 3 (1951) 391-441. MR0044580 $(13: 443 \mathrm{c})$

[DG95] M. Deza, V. Grishukhin, Delaunay polytopes of cut lattices, Linear Algebra Appl. 226/228 (1995) 667-685. MR1344592 (96f:52021)

[DL97] M. Deza, M. Laurent, Geometry of cuts and metrics, Springer, 1997. MR1460488 (98g:52001)

[DKS03] I. Dinur, G. Kindler, S. Safra, Approximating CVP to within almost-polynomial factors is NP-hard, Combinatorica 23 (2003), 205-243. MR2001908 (2004h:68046)

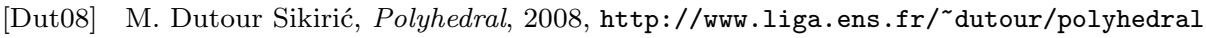

[DSV07] M. Dutour Sikirić, A. Schürmann, F. Vallentin, Classification of eight-dimensional perfect forms, Electron. Res. Announc. Amer. Math. Soc. 13 (2007) 21-32. MR2300003 (2007m:11089)

[Dy83] M. Dyer, The complexity of vertex enumeration methods, Math. Oper. Res. 8 (1983) 381-402. MR716120 (85j:68102)

[Ede01] H. Edelsbrunner, Geometry and topology for mesh generation, Cambridge University Press, 2001. MR1833977 (2002k:65206)

[EMS03] P. Engel, L. Michel, M. Sénéchal, Lattice geometry, preprint, 2003.

[ELZ05] U. Erez, S. Litsyn, R. Zamir, Lattices which are good for (almost) everything, IEEE Trans. Inform. Theory 51-10 (2005) 3401-3416. MR2236418 (2007a:94129)

[FR05] L. Fukshansky, S. Robins, Frobenius problem and the covering radius of a lattice, Discrete Comput. Geom. 37 (2007), 471-483. MR2301530(2008a:11040)

[Fuk95] K. Fukuda, cdd+ reference manual, Institute for operations research, Swiss Federal Institute of Technology, Zurich, Switzerland, 1995, http://www.ifor.math.ethz.ch/ fukuda/cdd_home/cdd.html.

[GG92] A. Gersho, R. M. Gray, Vector Quantization and Signal Processing, Kluwer Academic Press/Springer, 1992.

[GZ83] C. Greene, T. Zaslavsky, On the interpretation of Whitney numbers through arrangements of hyperplanes, zonotopes, non-Radon partitions, and orientations of graphs, Trans. Amer. Math. Soc. 280 (1983), 97-126. MR712251 (84k:05032)

[GMR05] V. Guruswami, D. Micciancio, O. Regev, The complexity of the covering radius problem, Comput. Complexity 14 (2005) 90-121. MR2189919 (2006k:68041)

[HR06] I. Haviv, O. Regev, Hardness of the covering radius problem on lattices, pages 145-158 in Proc. of 21st IEEE Annual Conference on Computational Complexity (CCC), 2006.

[KBBEG08] L. Khachiyan, E. Boros, K. Borys, K. Elbassioni, V. Gurvich, Generating all vertices of a polyhedron is hard, Discrete Comput. Geom. 39 (2008) 174-190. MR2383757

[KST93] J. Köbler, U. Schöning, J. Torán, The graph isomorphism problem: its structural complexity, Birkhäuser, 1993. MR.1232421 (95b:05154)

[Las98] J. B. Lasserre, Integration on a convex polytope, Proc. Amer. Math. Soc. 126 (1998) 2433-2441. MR.1459132 (98j:65016)

[Lin86] N. Linial, Hard enumeration problems in geometry and combinatorics, SIAM J. Algebraic Discrete Methods 7 (1986) 331-335. MR830652 (87e:68029)

[Mar97] A. Marzetta, pd-C-implementation of the primal dual algorithm, 1997, http://www. cs.unb.ca/profs/bremner/pd/.

[McK06] B. D. McKay, nauty User's Guide (Version 2.4), 2006, http://cs.anu.edu.au/ people/bdm/nauty/nug-2.4b3.pdf.

[Mic04] D. Micciancio, Almost perfect lattices, the covering radius problem, and applications to Ajtai's connection factor, SIAM J. Comput. 34 (2004) 118-169. MR2114308 (2005h:68049)

[MP95] R.V. Moody, J. Patera, Voronoi domains and dual cells in the generalized kaleidoscope with applications to root and weight lattices, Canad. J. Math. 47 (1995) 573-605. MR.1346154 (97c:17008)

[OPS98] J. Opgenorth, W. Plesken, T. Schultz, Crystallographic algorithms and tables, Acta Cryst. Sect. A 54 (1998) 517-531. MR1645546 (99h:20082)

[Ox192] J. G. Oxley, Matroid theory, Oxford University Press, 1992. MR1207587 (94d:05033)

[PP97] W. Plesken, B. Souvignier, Computing isometries of lattices, J. Symbolic Comput. 24 (1997) 327-334. MR1484483 (98i:11047) 
[Roc70] R. T. Rockafellar, Convex analysis, Princeton University Press, 1970. MR0274683 $(43: 445)$

[Sch86] A. Schrijver, Theory of linear and integer programming, Wiley, 1986. MR874114 (88m:90090)

[SV06] A. Schürmann, F. Vallentin, Computational approaches to lattice packing and covering problems, Discrete Comput. Geom. 35 (2006) 73-116. MR2183491 (2006k:52048)

[SB03] N. J. A. Sloane, B. Beferull-Lozano, Quantizing using lattice intersections, Discrete and computational geometry, 799-824, Algorithms Combin., Springer, 2003. MR2038504 (2005b:52050)

[Tut71] W. T. Tutte, Introduction to the theory of matroids, American Elsevier Publishing Company, 1971. MR0276117 (43:1865)

[VB96] E. Viterbo, E. Biglieri, Computing the Voronoi cell of a lattice: the diamond-cutting algorithm, IEEE Trans. Inform. Theory 42 (1996) 161-171. MR.1375332 (96j:68177)

[Vor08] G. F. Voronoi, Nouvelles applications des paramètres continus à là théorie des formes quadratiques, Deuxième Mémoire, Recherches sur les parallélloedres primitifs, J. Reine Angew. Math. 134 (1908) 198-287 and 136 (1909) 67-181.

[Zie95] G. M. Ziegler, Lectures on polytopes, Springer, 1995. MR1311028 (96a:52011)

Rudjer Bosković Institute, Bijenicka 54, 10000 Zagreb, Croatia

E-mail address: mdsikir@irb.hr

Mathematics Department, University of Magdeburg, 39106 Magdeburg, Germany

E-mail address: achill@math.uni-magdeburg.de

Centrum voor Wiskunde en Informatica (CWI), Kruislaan 413, 1098 SJ Amsterdam, The NetherLands

E-mail address: f.vallentin@cwi.nl 